\title{
Solution method using correlated noise for TSP
}

\author{
Atsuko Goto and Masaki Kawamura \\ Yamaguchi University, 1677-1 Yoshida, Yamaguchi, Japan \\ \{kawamura, atsuko21\}@is.sci.yamaguchi-u.ac.jp
}

\begin{abstract}
We suggest solution method for optimization problems using correlated noises. The correlated noises are introduced to neural networks to discuss mechanism of synfire chain. Kawamura and Okada have introduced correlated noises to associative memory models and have analyzed those dynamics. In the associative memory models, memory patterns are memorized as attractors in the minimum of the system. They found the correlated noise can make the state transit between the attractors. However, the mechanism of the state transition has not been known enough yet. One the other hand, for combinational optimization problems, the energy function of a problem can be defined. Therefore, finding a optimum solution is finding a minima of the energy function. The steepest descent method searches one of the solutions by going down along the gradient direction. By this method, however, the state is usually trapped in a local minimum of the system. In order to escape from the local minimum, the simulated annealing, i.e. Metropolis method, or chaotic disturbance is introduced. These methods can be represented by adding thermal noises or chaotic inputs to the dynamic equation.

In this paper, we show that correlated noises introduced to neural networks can be applied to solve the optimization problems. We solve the TSP that is a typical combinational optimization problem of NP-hard, and evaluated solutions obtained by using the steepest descent method, the simulated annealing and the proposed method with the correlated noises. As results, in the case of ten cities, the proposed method with correlated noises can obtain more optimum solutions than the steepest descent method and the simulated annealing. In the cases of large numbers of cites, where it is hard to find one of the optimum solutions, our method can obtain solutions at least as same level as the simulated annealing.
\end{abstract}

\section{Introduction}

In the activities of nerve cells, synfire chains, i.e. synchronous firings of neurons, can often be observed [1]. To analyze the mechanism of synchronous firings, condition for propagating them between layers have been investigated in layered neural networks $[2,3]$. In the layered neural networks, it has been proofed that the spacial correlation between neurons is necessary [4]. Aoki and Aoyagi [5] have 
shown that the state transition in associative memory models is invoked by not thermal independent noises but synchronous spikes. Kawamura and Okada [6] have proposed associative memory models to which common external inputs are introduced, and found that the state could transit between attractors by the inputs. The synchronous spikes of Aoki and Aoyagi model correspond to the common external inputs. In associative memory models, memory patterns are memorized as attractors. When we consider the energy function or cost function in the associative memory models, the attractors are represented by minimum of the system. The states of neurons are attracted into one of the memory patterns near the initial state.

A optimization problem is one of the problems to minimize the energy function. In engineering and social science, the optimization problems are important. The combinational optimization problem is one of the optimization problems, which is the problem that find the solution minimizing the value of object function in feasible area. Since number of feasible solutions is finite, some optimal solutions might be obtained when we could search all feasible solutions. Such solution methods are known as enumeration methods, i.e. branchand-bound method and dynamic programming. However, the combinational optimization problems are belonging to NP-hard, and then we cannot obtain solutions within a effective time by these methods. Therefore, instead of finding optimum solutions in whole feasible area, the methods that can find optimum or quasi-optimum solutions are developed. In these methods, the optimum solutions are designed as minimum of the energy function, and the problems are formulated as finding global minimum of the energy function. The steepest descent method (SDM), the simulated annealing (SA) [7$9]$, and chaotic method $[10,11]$ are introduced in order to find global minimum.

Since the steepest descent method obtains solutions along the gradient direction, the states cannot escape from local minimum. Therefore, thermal independent noise or chaotic noise is introduced to escape from the local minimum. The simulated annealing is the method using thermal independent noise. The optimum solution can be found by decreasing temperature $T$ through $T_{t+1} \geq c / \log (1+t)$, where $c$ is constant and $t$ represents time [9]. We consider the correlated noises introduced to associative memory models by Kawamura 
and Okada [6], since the correlated noises can make the state transit between attractors. The optimum and quasi-optimum solutions of the optimization problems can be assumed to be attractors, and it is expect that better optimum solutions can be easily obtained using the correlated noise. We, therefore, propose the method with the correlated noises in order to solve the combinational optimization problems. We can assume that the thermal noise used in the simulated annealing corresponds to independent noise, since the noise is fed to each element independently. The correlated noises that we propose is fed to all elements mutually. Therefore, the state of each element has spacial correlation. We show that the better solutions are obtained by the proposed method efficiently than the simulated annealing and the steepest descent method.

\section{TSP}

The traveling salesman problem, TSP, is one of the typical combinational optimization problems. The TSP is the problem that a salesman visits once each city and finds the shortest path. There are $(N-1) ! / 2$ different cyclic paths for $N$ cities. In this paper, we show that the correlated noise can be applied to the combinational optimization problem. This kind of problems is formulated as the problem for which one obtains minimum values of its energy function. The state variable $V_{x i}$ takes 1 when a salesman visits $x$-th city at the $i$-th order, and 0 when he doesn't. The energy function of the TSP is defined as

$$
\begin{aligned}
E & =\alpha E_{c}+\beta E_{o}, \\
E_{c} & =\frac{1}{2} \sum_{x=1}^{N}\left(\sum_{i=1}^{N} V_{x i}-1\right)^{2}+\frac{1}{2} \sum_{i=1}^{N}\left(\sum_{x=1}^{N} V_{x i}-1\right)^{2} \\
& +\sum_{x=1}^{N} \sum_{i=1}^{N} V_{x i}\left(1-V_{x i}\right), \\
E_{o} & =\frac{1}{2} \sum_{x=1}^{N} \sum_{\substack{y=1 \\
y \neq x}}^{N} \sum_{i=1}^{N} \frac{d_{x y}}{\widetilde{d}} \frac{V_{x i} V_{y, i-1}+V_{x i} V_{y, i+1}}{2}
\end{aligned}
$$

where the energy $E_{c}$ and $E_{o}$ represent constrained condition and object function, respectively. The constant $d_{x y}$ represents distance 
between $x$-th and $y$-th cities, and the average distance $\tilde{d}$ is given by,

$$
\widetilde{d}=\frac{1}{N(N-1)} \sum_{x=1}^{N} \sum_{y=1}^{N} d_{x y},
$$

where it shows the average distance between all different cities. The coefficients $\alpha$ is usually $\alpha=1$, and $\beta$ is decided according to the cities' locations and the number of them. The optimum solutions are obtained by searching minimum of the energy function $E$. The minimum which shall be satisfied with $E_{c}=0$ are called solutions, and the the solutions which give the shortest paths are called optimum solutions.

\section{Proposed Method}

In order to obtain one of local minimum of the energy function $E$ by the steepest descent method, the state $V_{x i}(t)$ is updated by

$$
\begin{aligned}
\mu \frac{d u_{x i}(t)}{d t} & =-u_{x i}(t)+\sum_{y=1}^{N} \sum_{j=1}^{N} W_{x i y j} V_{y j}(t)+\theta_{x i}, \\
V_{x i}(t) & =F\left(u_{x i}(t)\right),
\end{aligned}
$$

where the function $F$ is the output function which decides output $V_{x i}(t)$ according to the internal state $u_{x i}(t)$. We used the output function,

$$
F(u)= \begin{cases}1, & 1<u \\ u, & 0<u \leq 1 \\ 0, & u \leq 0\end{cases}
$$

From the energy function, the constant $W_{x i y j}$ is given by

$$
W_{x i y j}=-\delta_{x, y}\left(1-\delta_{i, j}\right)-\delta_{i, j}\left(1-\delta_{x, y}\right)-\beta \frac{d_{x y}}{\widetilde{d}}\left(\delta_{i-1, j}+\delta_{i+1, j}\right)\left(1-\delta_{x, y}\right),
$$

and the external input $\theta_{x i}$ is constant $\theta_{x i}=1$. The delta function $\delta_{x, y}$ is defined as

$$
\delta_{x, y}=\left\{\begin{array}{l}
1, x=y \\
0, x \neq y
\end{array} .\right.
$$


When independent noise $\zeta_{x i}(t)$ is introduced to (5), the equation corresponds to the simulated annealing. When correlated noise $\eta(t)$ is introduced, the equation gives the proposed method. Therefore, we consider the equation given by

$$
\begin{aligned}
\mu \frac{d u_{x i}(t)}{d t}= & -u_{x i}(t)+\sum_{y=1}^{N} \sum_{j=1}^{N} W_{x i y j} V_{y j}(t)+\theta_{x i} \\
& +\zeta_{x i}(t)+\eta(t), \\
V_{x i}(t)= & F\left(u_{x i}(t)\right) .
\end{aligned}
$$

We note that independent noise $\zeta_{x i}(t)$ is fed to each neuron independently, and the correlated noise $\eta(t)$ is fed to all neurons mutually. We assume that the independent noise obeys normal distribution with mean 0 and variance $\sigma_{\zeta}^{2}$, and the correlated noises obeys normal distribution with mean 0 and variance $\sigma_{\eta}^{2}$.

\section{Simulation Results}

\section{1 locations of cities}

Figure 1 shows the locations for 10 cities that are arranged in random order, and one for 29 cities named bayg29 in TSPLIB [12]. The shortest path for which a salesman visits 10 cities is -A-D-B-E-J-HI-G-F-C-, and for 29 cities -1-28-6-12-9-26-3-29-5-21-2-20-10-4-15-1814-17-22-11-19-25-7-23-8-27-16-13-24-. The distance of the shortest path of 10 cities is 2.69, and one of 29 cities is 9074.15 , where the significant figure is until second decimal place.

\section{2 experimental procedure}

The initial values of internal state $u_{x i}(t)$ are determined at random with uniform distribution on the interval $[-0.01,0.01)$. Using the steepest descent method, the method with independent noises, and proposed method, we perform computer simulations where $\alpha=1$ in (1). For the case of the 10 cities, we perform this case 100 times, and for the case of the 29 cities, 1000 times.

We evaluate the ratio $R$ of the path length for an obtained solution to the optimum path length,

$$
R=\frac{[\text { path length for obtained solution }]}{[\text { optimum path length }]} \text {. }
$$




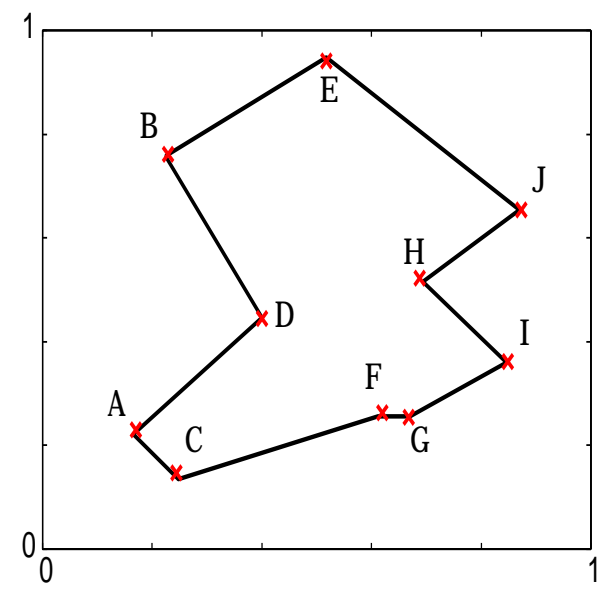

(a) 10 cities

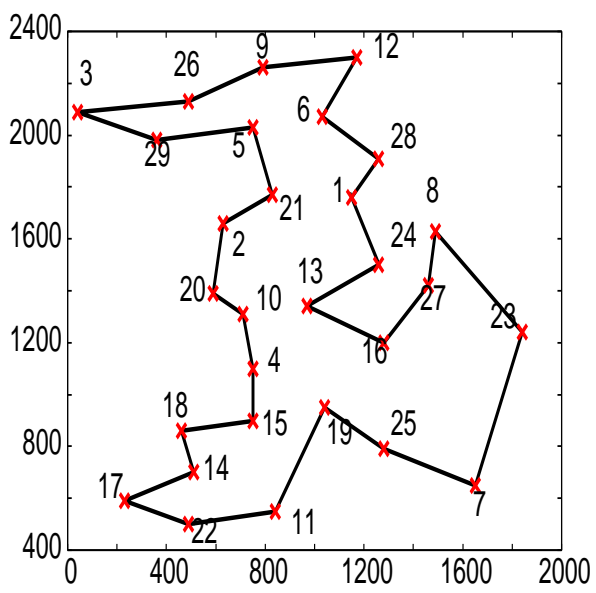

(b) 29 cities

Fig. 1. Locations of (a) 10 cities and (b) 29 cities. The paths show the optimal solutions.

Since the state must satisfy $0-1$ condition when the state converges, the final state is given by,

$$
V_{x i}=H\left(u_{x i}\right),
$$

where the function $H\left(u_{x i}\right)$ is given by,

$$
H\left(u_{x i}\right)=\left\{\begin{array}{ll}
1, & u_{x i}>0 \\
0, & u_{x i} \leq 0
\end{array} .\right.
$$

\section{3 results}

For the 10 cities, we assume $\beta=0.35$, the variance of independent noise is $\sigma_{\zeta}^{2}=0.08$, and the variance of correlated noise is $\sigma_{\eta}^{2}=0.08$. We calculate the number of optimum solutions on 100 trials for this location. The histogram of the path length, when we can obtain solutions, is shown in Fig.2. Abscissa represents the ratio $R$ in (12), and ordinate represents the percentage of number of ratio $R$. The number of obtained solutions by the steepest descent method is 17 times, that by the method with independent noise is 55 times, and that by proposed method with correlated noises is 90 times. The proposed method can obtain most solutions in these methods. Next, 


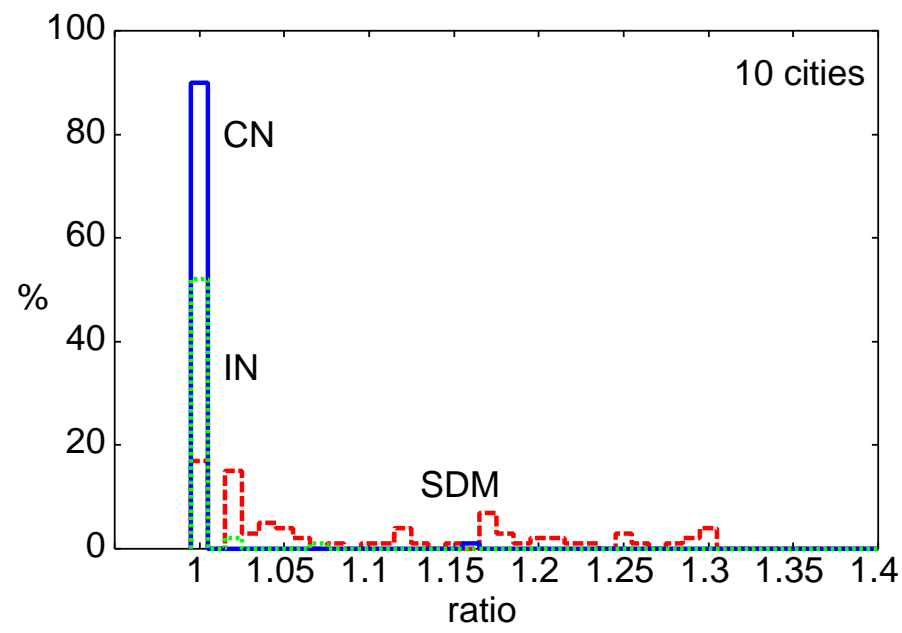

Fig. 2. Histogram of path length of obtained solutions for 10 cities. Solid, dot, and broken lines represent results obtained by correlated noise $(\mathrm{CN})$, independent noise (IN), and steepest decent method (SDM), respectively.

Figure 3 shows the transition of residual energy by the method with independent noise and correlated noise, where the updating steps of internal state are 1,000,000 times. The residual energy $E_{\text {res }}$ means difference between energy $E(t)$ of $V_{x i}$ at time $t$ and the energy of the optimum solution, $E_{\text {opt }}$;

$$
E_{\mathrm{res}}=E(t)-E_{\mathrm{opt}} \cdot
$$

We found that the energy do not go down through 0 by the method with independent noise, but by the proposed method.

For the 29 cities, we calculate the solutions, where the variances of independent noise are $\sigma_{\zeta}^{2}=0.01 \sim 0.10$, and the variances of correlated noise are $\sigma_{\eta}^{2}=0.01 \sim 0.10$. The optimum solutions could not be obtained by all these methods for this location. Therefore, we calculate the path lengths for obtained solutions. Figure 4 shows the histogram of path lengths when solutions are obtained. The abscissa represents the ratio $R$, and the ordinate represents the percentage that the solutions having ratio $R$ are obtained. The method with independent noise and the proposed method can obtain better solutions than the steepest descent method. 


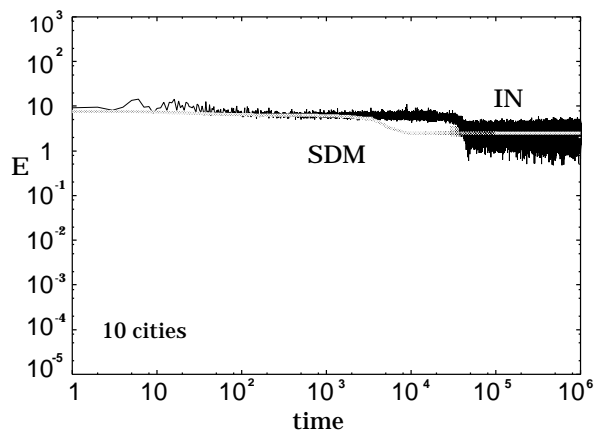

(a)

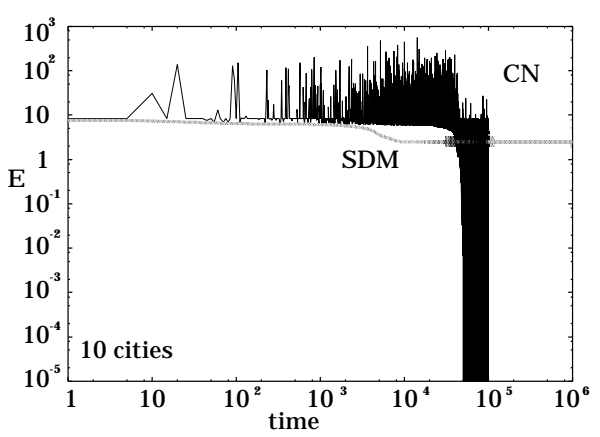

(b)

Fig. 3. Residual energy by (a) independent noises (IN) and (b) correlated noise (CN) for 10 cities.

Table 1. The optimum variance and the solution ratio for $\beta=0.35$ and $\beta=0.5$.

\begin{tabular}{|l|c|c|c|c|}
\hline & \multicolumn{2}{|c|}{$\beta=0.35$} & \multicolumn{2}{c|}{$\beta=0.5$} \\
\hline optimum variance & $\sigma_{\zeta}^{2}=0.03$ & $\sigma_{\eta}^{2}=0.03$ & $\sigma_{\zeta}^{2}=0.05$ & $\sigma_{\eta}^{2}=0.05$ \\
\hline solution ratio(\%) & 93 & 98 & 56 & 75 \\
\hline
\end{tabular}

We compare the solution ratio of obtained solutions for the method with independent noise with one for the proposed method. Figure 5 shows the solution ratio for variances $\sigma_{\zeta}^{2}$ and $\sigma_{\eta}^{2}$ in the cases of $\beta=0.35,0.50$. Table 1 shows the optimum variance and the solution ratio. In the case of $\beta=0.35$, the number of obtained solutions by the method of independent noises with $\sigma_{\zeta}^{2}=0.03$ is 93 times. The number of obtained solutions by the proposed method with $\sigma_{\eta}^{2}=0.03$ is 98 times. There are not so much of a difference between them. On the other hand, in the case of $\beta=0.5$, number of solutions by the method of independent noises with $\sigma_{\zeta}^{2}=0.05$ is 56 times and, one by the proposed method with $\sigma_{\eta}^{2}=0.05$ is 75 times. Namely, the proposed method can obtain better solutions than the method with independent noises. We, therefore, found that the proposed method can be much more effective than the method with independent noises in order to obtain solutions depending on $\beta$. 


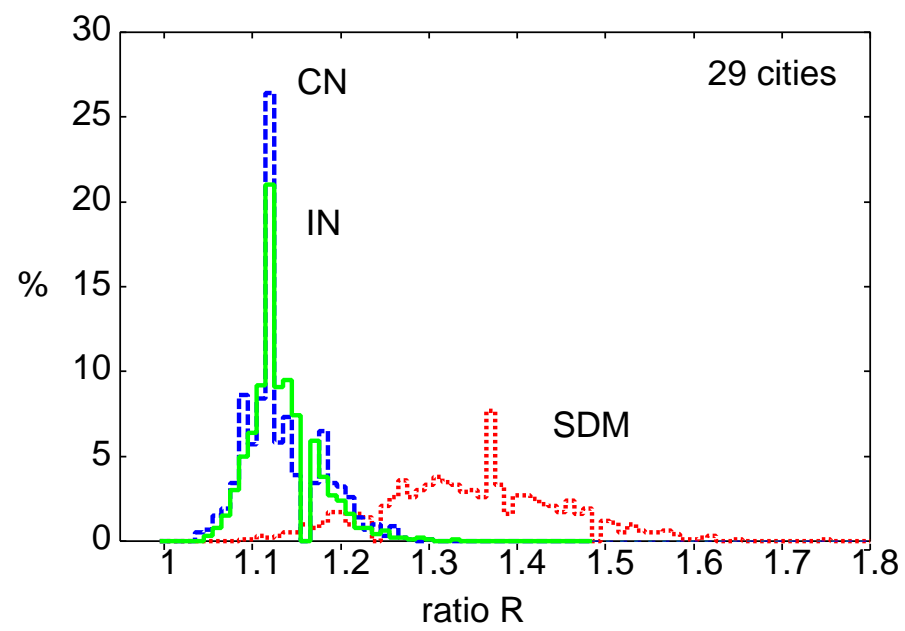

Fig. 4. Histogram of path length for 29 cities. we assumed $\beta=0.35$, the variance of independent noise is $\sigma_{\zeta}^{2}=0.04$, and the variance of correlated noise is $\sigma_{\eta}^{2}=0.04$. The number of obtained solutions by the CN is 979 times, one by IN is 931 times and one by the SDM is 883 times.

\section{Conclusion}

In associative memory models, the correlated noise is effective in state transition. In this paper, we proposed the solution method using the correlated noise and applied to TSP that is one of the typical combinational optimization problems of NP-hard. As the results, for the case of the 10 cities, the proposed method with the correlated noises can obtains more solutions than both the steepest descent method and the method with independent noises. For the case of the 29 cities, all these methods cannot be obtained any optimum solutions. However, we found that the proposed method can obtain better solutions than the existing methods depending on $\beta$. From these results, we can show that the correlated noises is also effective for the combinational optimization problems.

\section{acknowledgments}

This work was partially supported by a Grant-in-Aid for Young Scientists (B) No. 16700210. The computer simulation results were obtained using the PC cluster system at Yamaguchi University. 


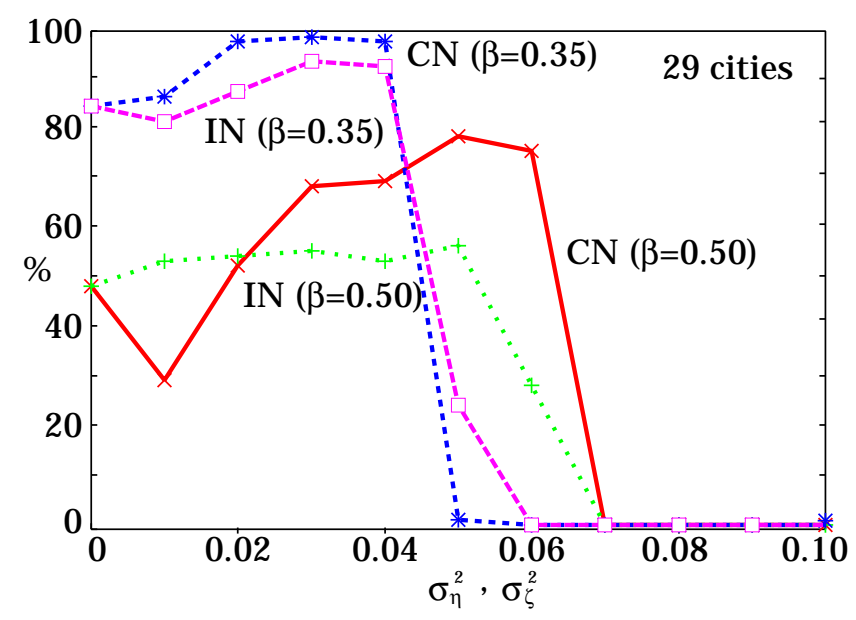

Fig. 5. Solutions ratio (\%) for 29 cities.

\section{References}

1. Abeles, M. : Corticonics, Cambridge Univ. Press, Cambridge (1991)

2. Dlesmann, M., Gewaltig, M.-O., and Aertsen, A. : Stable Propagation of Synchronous Spiking in Cortical Neural Networks, Nature, Vol. 402 (1999) 529-533

3. Câteau, H., and Fukai, T. : Fokker-Planck Approach to the Pulse Packet Propagation in Synfire Chain, Neural Networks, Vol. 14 (2001) 675-685

4. Amari, S., Nakahara, H., Wu, S., and Sakai, Y. : Synchronous Firing and HigherOrder Interactions in Neuron Pool, Neural.Comp., Vol. 15 (2003) 127-143

5. Aoyagi, T., and Aoki, T. : Possible Role of Synchronous Input Spike Trains in Controlling the Function of Neural Networks, Neurocomputing, Vol. 58-60 (2004) $259-264$

6. Kawamura, M., and Okada, M. : Stochastic Transitions of Attractors in Associative Memory Models with Correlated Noise, J. Phys. Soc. Jpn, Vol. 75 (2006) 124603

7. Kirkpatrick, S., Gelatt, C. D., and Vecchi, M. P. : Optimization by Simulated Annealing, Science, Vol. 220 (1983) 671-680

8. Metropolis, N., Rosenbluth, A, Rosenbluth, M, Teller, A, and Teller, E.: Equation of State Calculations by Fast Computing Machies, J. Chem. Phys., Vol. 21, No.6 (1953) 1087-1092

9. Geman, S., and Geman, D. : Stochastic Relaxation, Gibbs Distributions, and the Bayesian Ryestoration of Image, IEEE Trans. Patt. Anal. Mach. Intel., Vol. PAMI6 (1984) 721-741

10. Zhou, C-S., and Chan, T-L. : Chaotic Annealing for Optimization, Phys. Rev. E, Vol. 55 (1997) 2580-2587

11. Tokuda, I., Nagashima, T., and Aihara, K. : Global Bifurcation Structure of Chaotic Neural Networks and its Application to Traveling Salesman Problems, Neural Networks, Vol. 10 (1997) 1673-1690

12. TSPLIB http://www.iwr.uni-heidelberg.de/groups/comopt/software/TSPLIB95/ 